\title{
Reflets
}

Revue ontaroise d'intervention sociale et communautaire

\section{Osez parler, osez découvrir, osez s’affirmer}

\section{Jean-Marc Bélanger et Richard Carrière}

Volume 4, numéro 2, automne 1998

Personnes vivant avec une incapacité

URI : https://id.erudit.org/iderudit/026214ar

DOI : https://doi.org/10.7202/026214ar

Aller au sommaire du numéro

Éditeur(s)

Reflets : Revue ontaroise d'intervention sociale et communautaire

ISSN

1203-4576 (imprimé)

1712-8498 (numérique)

Découvrir la revue

Citer ce document

Bélanger, J.-M. \& Carrière, R. (1998). Osez parler, osez découvrir, osez s'affirmer. Reflets, 4(2), 10-17. https://doi.org/10.7202/026214ar

Tous droits réservés (C) Reflets : Revue ontaroise d'intervention sociale et communautaire, 1998
Ce document est protégé par la loi sur le droit d'auteur. L'utilisation des services d'Érudit (y compris la reproduction) est assujettie à sa politique d'utilisation que vous pouvez consulter en ligne.

https://apropos.erudit.org/fr/usagers/politique-dutilisation/ 


\section{Osez parler, osez découvrir, osez s'affirmer}

\section{Jean Marc Bélanger et Richard Carrière}

Ce huitième numéro de la revue Reflets lève le voile sur un thème quasi absent dans les écrits en Ontario français, et peu fréquent ailleurs au Canada. Il vous invite à explorer les besoins, les défis, les aspirations et les réalisations de personnes ayant une incapacité.

Parler d'incapacité touche les cordes sensibles de plusieurs personnes, tant chez celles qui vivent quotidiennement avec une incapacité que chez celles qui ont à intervenir directement ou indirectement auprès d'elles. En effet, on ne peut pas parler des personnes ayant une incapacité, sans rappeler les diverses connotations péjoratives dont elles furent l'objet.Trop longtemps les étiquettes d'infirme ou d'invalide ont stigmatisé et ont signifié leur exclusion sociale. On les enfermait ou on les cachait à la maison. Grâce, en partie, aux luttes anti-discriminatoires et aux revendications en faveur de l'équité, ce n'est que tout récemment que la société leur a ouvert les portes. Les questions d'accessibilité et d'intégration sont devenues des aspects importants de l'agenda social de nos gouvernements.

Un des premiers signes de ce changement d'attitudes envers les personnes ayant une incapacité est apparu dans le langage. On a rejeté les anciens termes à la faveur d'une nouvelle terminologie qui soulignait à la fois la valeur de la personne, tout en reconnaissant ses besoins.Ainsi, est née l'expression contemporaine de «personne ayant une incapacité». Comme toute personne, nous sommes des êtres de besoins, mais nos capacités d'y répondre varient énormément que ce soit au plan biologique, psychologique, social ou culturel. Changer le langage ne permettra pas à lui seul d'enrayer 
l'image stéréotypée associée aux personnes ayant une incapacité. Mais il peut être le signe avant-coureur d'une plus grande ouverture à leur parole. C'est dans cet esprit que nous avons créé, pour ce numéro, une rubrique spéciale intitulée Témoignages. Si on ne donne pas la parole aux personnes ayant une incapacité, il sera impossible de connaitre leurs besoins, de les connaitre et d'apprécier leurs contributions. Trop longtemps on a parlé d'elles et pour elles, il est temps maintenant de leur donner la parole. En lisant ces témoignages, vous découvrirez chez nos collaborateurs et nos collaboratrices leur capacité nourrie à même un courage, une volonté d'agir, un désir de réussir pleinement sa vie et un sens d'accomplissement sans pareil. Tout se passe comme si la capacité naissait de l'incapacité, puisqu'elle demande d'aller audelà de nos limites physiques ou mentales, pour rejoindre nos sources intérieures, les approfondir et y puiser la persévérance et la volonté de grandir. En ce sens, les capacités dont elles font preuve sont peut-être moins une destination qu'une invitation au voyage. Dans ce voyage, nous nous apercevrons que nos jugements coupent souvent les ailes à leur envolée, en le renvoyant au cadre social de l'incapacité. Ce n'est là qu'une des barrières mentionnées dans ce numéro de Reflets qui vous présente des articles de gens qui réfléchissent sur la notion de l'incapacité, qui rendent visibles les besoins liés à leur volonté d'actualiser leurs capacités et qui examinent certains services et programmes disponibles pour appuyer les personnes dans leur cheminement d'intégration.

Stéphane Laberge, dans la section Entrevue, présente l'expérience de deux femmes qui, avec l'appui du Centre de participation communautaire, se sont prises en main pour affirmer leur existence. Elles ont participé à la production d'une vidéo promotionnelle des activités du Centre qui leur a permis de mettre en valeur leurs capacités. Dans cette entrevue, Stéphane fait ressortir l'importance d'organismes comme le Centre de participation communautaire qui permet à ces personnes de s'exprimer et d'élaborer des projets.

Dans la section Dossiers, nous présentons des textes qui montrent les limites imposées par la société. Le texte de Burelle 
porte sur l'intégration des personnes ayant une incapacité.D'abord, elle trace à grands traits l'historique des politiques sociales, en insistant plus particulièrement sur l'eugénisme qui est au fondement de l'institutionnalisation des personnes ayant une incapacité. Elle aborde ensuite l'ère du renouveau, axée sur un principe d'intégration, qui s'instaure au tournant des années quatre-vingts avec cet événement repère qu'est l'année internationale des handicapés. En Ontario, on verra naitre à la même époque, l'Office des personnes handicapées, qui adhèrent à ce même principe.

Sans renoncer à ce principe, le gouvernement ontarien déposait dernièrement le projet de loi 142, la Loi de 1997 sur la réforme de l'aide sociale. Cette mesure législative comprend deux volets distincts, soit la loi de 1997 sur le programme Ontario au travail (LPOT) et la loi de 1997 sur le Programme ontarien de soutien aux personnes handicapées (LPOSPH $)^{1}$. À son tour, cette dernière se subdivise en deux parties. La première touche les allocations financières, alors que la seconde a trait aux services de support à l'emploi.

Outre l'objectif de scinder la clientèle de l'aide sociale entre aptes au travail et inaptes, on peut reprocher à cette réforme, dans son volet "Programme de soutien aux personnes handicapées", d'adopter une définition restreinte de ce qu'est une personne handicapée. Selon le groupe Advocacy Resource Centre for the Handicapped (ARCH), cette réforme fera en sorte que moins de personnes se qualifieront pour répondre aux exigences de cette définition et ainsi, moins de personnes seront admissibles au nouveau programme de soutien du revenu. Dans son volet «services de soutien à l'emploi», on semble mettre indûment l'emphase sur l'individu. L'intégration est dorénavant du ressort de l'individu. Cette accentuation masque le fait qu'aucune ressource supplémentaire ne soit allouée pour faciliter l'accessibilité des personnes handicapées au marché du travail ou du moins, d'améliorer leurs chances d'y accéder plus facilement. En fait, on néglige les obstacles systémiques que rencontrent une personne ayant une incapacité et on fait reposer l'effort d'intégration sur ses épaules. C'est d'ailleurs sur cette note, celle des obstacles 
systémiques, que se termine le texte de Burelle, en affirmant que la lutte menée aujourd'hui n'est plus contre l'exclusion, mais pour l'intégration. Cette intégration passe par l'implication des personnes ayant une incapacité dans l'élaboration des politiques sociales les concernant.

L'article de Barette, Laroche et Garcia porte sur les troubles de la communication et les obstacles au travail. Les auteurs présentent à la fois les défis rencontrés par les personnes ayant une incapacité et ceux des organisations. Les personnes aux prises avec des troubles de la communication doivent souvent trouver par elles-mêmes les stratégies pour contourner les obstacles qui les empêchent d'être pleinement actifs dans le monde du travail. Par ailleurs, les employeurs et les organismes se doivent d'offrir et de créer un environnement de travail exempt de barrières discriminantes. En établissant un outil de travail permettant d'évaluer l'environnement de travail, ces auteurs montrent que la réintégration au travail est un défi devant être partagé également entre les intervenants professionnels, les employeurs et les personnes vivant une incapacité.

Dans son article, Marc Charron communique les résultats d'une recherche-action menée par les Sourds francophones du Nordest ontarien. L'objet de cette recherche visait à comprendre la culture sourde. Dans cette recherche, l'auteur établit une comparaison entre les Sourds et les malentendants, en précisant qu'une personne qui se dit Sourde s'affiche comme appartenant à la culture Sourde, et cette appartenance n'a rien à voir avec le degré de surdité ou le niveau de décibels qu'un individu peut ou ne peut pas entendre. Outre cette revendication d'appartenance, Charron note qu'un des traits qui distingue les Sourds des malentendants est le degré de participation à la culture entendante. En effet, les personnes malentendantes participent davantage à la culture entendante que les Sourds et cela se manifeste dans l'utilisation du français comme langue d'usage chez la majorité des personnes malentendantes, contrairement aux Sourds qui indiquent, comme langue d'usage, la LSQ (Langue des signes québécoise). 
Pour terminer notre rubrique Dossier, Louise Blais et Louise Mulligan-Roy présentent les résultats préliminaires d'une recherche portant sur le thème de l'incapacité mentale vue à travers la lorgnette de nos propres incapacités sociales. Ces auteures affirment «qu'être déclaré «incapable mental» aux termes de la Loi sur l'incapacité mentale, n'est pas du tout synonyme d'incapacité à comprendre ce qui nous arrive, de donner sens à notre expérience ou d'élaborer des activités permettant de survivre malgré ou avec des difficultés énormes». Leur recherche fera donc place à cette mise en parole et à la construction du sens des personnes psychiatrisées. Les auteures montrent que l'exclusion dont souffrent les personnes psychiatrisées est la conséquence d'une série de rejets sociaux, comme la pauvreté, de liens sociaux fragiles, la stigmatisation, qui les poussent toujours plus à la marge. Loin de s'en tenir à ce seul constat, les auteures ont également identifié les stratégies adoptées par les «incapables mentaux» pour combattre les contraintes de leur quotidien.

La rubrique Pratiques à notre image traite d'une grande variété de services offerts aux personnes ayant une incapacité en Ontario et examine certaines pratiques ou recherches les concernant.Tout d'abord, Danielle Perron-Roach s'interroge sur l'approche institutionnelle et invite les intervenantes et les intervenants à réfléchir à la façon dont nous intervenons auprès de personnes ayant un handicap. Pour ce faire, elle illustre son propos à l'aide d'une étude de cas. Elle y dénonce l'approche institutionnelle qui trop souvent donne préséance à nos connaissances et nos perceptions d'«experts» sur la façon dont le client ou la cliente se perçoit et négligent de voir son habileté à se prendre en charge. L'article de Ann Toth porte sur la communication avec la population des Sourds et des entendants. Elle présente un modèle de communication qui se veut respectueux des différences linguistique et culturelle entre les Sourds et les entendants. Ce modèle promeut la communication saine et la résolution de problème. À l'aide de ce modèle, elle souhaite ériger un pont entre les Sourds et les entendants. Le texte de Louise GervaisGuy,Jean Lafond et Denise Bellehumeur décrit le modèle utilisé par le Collège Boréal pour desservir la clientèle étudiante 
francophone ayant des besoins particuliers. Les auteurs donnent un aperçu du contexte unique où se trouve le Collège Boréal, discutant de sa technologie avant-gardiste, de son approche humaniste, ainsi que des services offerts à la population ayant des besoins particuliers. La recherche de Lynn Lambert aborde la question du mauvais traitement envers les femmes ayant un handicap, afin de sensibiliser les professionnels de la santé à ce sujet. La première étape de la recherche a consisté en une analyse de leurs besoins et la collecte des expériences vécues par les femmes ayant un handicap. En plus de présenter un portrait sociodémographique de ces femmes, Lambert relate leurs expériences auprès des professionnels de la santé. L'auteure termine son texte en soulignant la difficulté que peut représenter l'approche d'un tel sujet. Le texte de Lise Nolet traite de la sclérose en plaques. Après en avoir donné une définition, elle discute des conséquences sociales et financières de cette maladie, pour ensuite discuter des traitements disponibles et des soins de longue durée. L'auteure évoque le rôle de la Société de la sclérose en plaques et discute quelques-uns des défis qui attendent les personnes souffrant de sclérose en plaques à l'avenir. Pour conclure cette rubrique, Jean-Roger Brisebois discute de l'intervention auprès de personnes ayant une perte auditive. En insistant sur le fait que la perte auditive est un handicap invisible, l'auteur décrit son expérience de malentendant et l'attention particulière dont cette incapacité doit être l'objet. Il présente les divers services offerts aux personnes malentendantes dans la région de Sudbury, et montre l'importance pour les intervenantes et les intervenants de parvenir à établir un mode de communication préalable avec la personne sourde, devenue sourde ou malentendante avant de poursuivre l'intervention.

Sous la nouvelle rubrique Témoignages, nous vous présentons six témoignages de personnes qui ont raconté le sens qu'elles donnent à l'incapacité, soit pour en être atteintes elles-mêmes, soit d'accompagner des personnes qui en sont atteintes. C'est avec beaucoup de reconnaissance que nous remercions Fernande Rancourt, John McDonell, Nicole Rioux, Ronald, Fleur-Ange Rancourt et Suzanne Fortin d'avoir bien voulu partager leurs réussites, leurs espoirs. 
La rubrique Aux quatre coins vous renseignera sur différents services répartis un peu partout dans la province et pouvant venir en aide aux personnes vivant avec une incapacité. Hélène Gagné présente la nouvelle Fondation de neurotraumatologie à Toronto, pour les personnes victimes d'une lésion cérébrale ou d'un traumatisme rachidien. Carmelle Dugas parle de la Maison la Paix qui vient en aide aux personnes atteintes du sida. Noël Thomas s'interroge sur le rôle de l'Internet et des nouvelles technologies de la communication. Il se demande si ces nouveaux moyens constituent une nouvelle ressource ou nouvelle barrière pour les personnes ayant une incapacité. Souhaitant ne pas répéter les erreurs du passé, il invite le personnel technique et le personnel des relations humaines à être sensibles à cette problématique. Carole Groulx aborde le modèle de coordination des services duYWCA de Sudbury, qui est un modèle de prestation de services autonomes où les coordonnatrices de services peuvent intervenir selon les préférences et les besoins exprimés par la clientèle ayant une incapacité. Smail Gounane présente le journal Défi qui a été créé afin de combattre l'ignorance et favoriser une meilleure compréhension de la situation des personnes handicapées. Diane Vachon se fait le porte-parole de RESO, un regroupement des parents et d'amis des enfants sourds et malentendants francoontariens. Alain Poirier et Monique Morin-Lavigne décrivent une journée typique dans le programme du P'tit Bonheur du Patro d'Ottawa, un programme destiné aux personnes ayant un handicap intellectuel. Cette rubrique se termine par un résumé des activités du Centre franco-ontarien de ressources en alphabétisation (Centre FORA), qui depuis sa création en 1989 a participé à au-delà de 130 productions. Il souligne l'apport du Centre Fora qui vient en aide aux personnes éprouvant des difficultés d'apprentissage.

En terminant, nous vous invitons à lire la section Lu pour vous où Rachid Bagaoui, Richard Carrière, Madeleine Dubois et Marie-Luce Garceau résument quatre documents d'actualité touchant le domaine de l'incapacité et la réforme du système de santé. En dressant une bibliographie des Publications récentes se rapportant à l'incapacité, Lorraine Albert, comme nous tous, 
devons nous rendre à l'évidence qu'il y a très peu d'écrits en Ontario français sur ce sujet. Comme intervenantes et intervenants, nous avons donc fort à faire pour combler cette lacune. Ce présent numéro de Reflets ouvre la porte. Nous vous souhaitons donc bonne lecture.

Note

1. Pour une revue critique du programme Ontario au travail, voir l'article de Chantal Cholette «Le travail obligatoire en Ontario: solution ou imposture?, Reflets, vol. 4, no 1, printemps 1998, 100-126. 